\title{
Structure of the Energy-Momentum Tensor and Applications
}

\section{Jonathan Hudson,}

Department of Physics, University of Connecticut, Storrs, CT 06269, USA

\section{Irina A. Perevalova,}

Physics Department, Irkutsk State University, Karl Marx str. 1, 664003, Irkutsk, Russia

\section{Maxim V. Polyakov,}

Petersburg Nuclear Physics Institute, Gatchina, 188300, St. Petersburg, Russia Institut für Theoretische Physik II, Ruhr-Universität Bochum, D-44780 Bochum, Germany

\section{Peter Schweitzer*}

Department of Physics, University of Connecticut, Storrs, CT 06269, USA

E-mail: peter.schweitzer@phys.uconn.edu

\begin{abstract}
The probably most fundamental information about a particle is contained in the matrix elements of its energy momentum tensor (EMT) which are accessible from hard-exclusive reactions via generalized parton distribution functions. The spin decomposition of the nucleon and Ji sum rule are one example. Less prominent but equally important information is encoded in the stress tensor, related to the spatial components of the EMT, which shows in detail how the strong forces inside the nucleon balance to form a bound state. This provides not only unique insights on nucleon structure. It also leads to fascinating new applications to hadron spectroscopy which allow us to formulate new interpretations of the charmonium-nucleon pentaquarks discovered by LHCb. Recent progress is reviewed in this short overview article.
\end{abstract}

QCD Evolution 2016

May 30-June 03, 2016

National Institute for Subatomic Physics (Nikhef), Amsterdam

\footnotetext{
* Speaker.
} 


\section{Introduction}

The matrix elements of the EMT define some of the most fundamental properties of a particle: mass, spin, and $D$-term. The first two are well-known properties, but the latter is not known for any particle. The nucleon EMT form factors are defined as [1]

$$
\left\langle p^{\prime}\left|\hat{T}_{\mu v}\right| p\right\rangle=\bar{u}^{\prime}\left[A(t) \frac{\gamma_{\mu} P_{v}+\gamma_{v} P_{\mu}}{2}+B(t) \frac{i\left(P_{\mu} \sigma_{v \rho}+P_{v} \sigma_{\mu \rho}\right) \Delta^{\rho}}{4 M_{N}}+D(t) \frac{\Delta_{\mu} \Delta_{v}-g_{\mu v} \Delta^{2}}{4 M_{N}}\right] u,
$$

with $P=\left(p+p^{\prime}\right) / 2, \Delta=\left(p^{\prime}-p\right), t=\Delta^{2}$ and nucleon spinors $\bar{u}^{\prime}=\bar{u}\left(p^{\prime}\right), u=u(p)$ normalized as $\bar{u} u=2 M_{N}$. Other notations include $M_{2}(t)=A(t), 2 J(t)=A(t)+B(t), D(t)=4 C(t)=\frac{4}{5} d_{1}(t)$. At zero momentum transfer the form factors satisfy the constraints

$$
A(0)=1, \quad B(0)=0, \quad D(0)=D=\text { unknown } .
$$

The first relation, interpreted in infinite momentum frame, means that nucleon's constituents carry its total momentum. The second, known as the vanishing of the nucleon's gravitomagnetic moment, is equivalent to $J(0)=\frac{1}{2}$ which means the nucleon's constituents carry its total angular momentum. The third relation in (1.2) defines what we mean by the $D$-term in this work.

The EMT form factors can be accessed via 2nd Mellin moments of unpolarized generalized parton distributions (GPDs) of quarks and gluons [2, 4, 3, 5, 6, 7]. This allows one to study the nucleon's spin decomposition [4], and also the "mass decomposition" can be addressed [8,9]. This information can be accessed through the quark and gluon contributions to $A(t)$ and $B(t)$.

But what exactly does $D(t)$ tell us about the nucleon? It is the purpose of this proceeding to highlight the physics associated with $D(t)$, and to discuss recent developments and applications.

\section{D-term, the last unknown global property}

The specific relation of the $D$-term to GPDs was clarified in [10]. (Notice that "our" $D(t)$ corresponds to the leading term in the Gegenbauer expansion of the " $D$-term" defined in [10].) Further aspects were discussed in $[6,11]$. Similarly to the way electric form factors provide insights on the electric charge distribution [12], EMT form factors offer insights on energy density $T_{00}(r)$, orbital angular momentum density, and stress tensor $T_{i j}(r)$ related to the $D$-term [13]. The interpretation is performed in the Breit-frame characterized by $\Delta^{0}=0$ such that 3D-Fourier transforms can be performed. This procedure is known to suffer from relativistic corrections [7], and justified only if the particle is heavy such that its Compton wavelength is much smaller than its "internal size." The nucleon is a heavy object in the limit of a large number of colors $N_{c}$ in QCD. But even in real life are the relativistic corrections small for the nucleon, and safely negligible for nuclei [14].

The $D$-terms of pions, nucleons and nuclei were investigated in a variety of theoretical approaches, including free field theories, soft-pion theorems, liquid drop model, chiral perturbation theory, bag model, chiral quark soliton model, lattice QCD, nuclear models, Skyrmions, and dispersion relations $[10,13,14,15,16,17,18,19,20,21,22,23,24,25,26,27,28,29,30,31,32]$. Also $D$-terms of photons [33], $Q$-balls and $Q$-clouds and their excitations [34, 35, 36, 37], and $\Delta$-resonances [38] were studied. In all theoretical approaches the $D$-terms of various particles were found negative. 
To understand why the $D$-term is the "last unknown global property" of the nucleon, we recall that the structure of strongly interacting particles is typically probed by means of the other fundamental forces: electromagnetic and weak interaction and, at least in principle, the gravity. The particles couple to the interactions via currents $J_{\mathrm{em}}^{\mu}, J_{\text {weak }}^{\mu}, T_{\mathrm{grav}}^{\mu v}$ which are conserved (in case of weak interactions we deal with the partial conservation of the axial current, PCAC). The matrix elements of these currents are described in terms of form factors which contain a wealth of information on the probed particle. The undoubtedly most fundamental information corresponding to the form factors at zero momentum transfer: the "global properties" electric charge $Q$, magnetic moment $\mu$, axial coupling constant $g_{A}$, induced pseudo-scalar coupling constant $g_{p}$, mass $M$, spin $J$, and $D$-term $D$. All global properties are well-known (see table below) and can be looked up e.g. in the particle data book, except for the $D$-term. See the following table for an overview.

\begin{tabular}{rlllrl}
\hline em: & $\partial_{\mu} J_{\mathrm{em}}^{\mu}=0$ & $\left\langle N^{\prime}\left|J_{\text {em }}^{\mu}\right| N\right\rangle$ & $\longrightarrow$ & $Q_{\text {prot }}$ & $=1.602176487(40) \times 10^{-19} \mathrm{C}$ \\
& & & $\mu_{\text {prot }}$ & $=2.792847356(23) \mu_{N}$ \\
\hline weak: & PCAC & $\left\langle N^{\prime}\left|J_{\text {weak }}^{\mu}\right| N\right\rangle$ & $\longrightarrow$ & $g_{A}$ & $=1.2694(28)$ \\
& & & & $g_{p}$ & $=8.06(0.55)$ \\
\hline gravity: & $\partial_{\mu} T_{\text {grav }}^{\mu v}=0$ & $\left\langle N^{\prime}\left|T_{\text {grav }}^{\mu \nu}\right| N\right\rangle$ & $\longrightarrow$ & $M_{\text {prot }}$ & $=938.272013(23) \mathrm{MeV} / c^{2}$ \\
& & & & $=$ \\
& & & $D$ & $=?$ \\
& & &
\end{tabular}

\section{3D densities of the EMT}

As shown in [13] performing the 3D Fourier-transforms of the form factors in Breit frame yields the components of static EMT $T_{\mu v}(\vec{r}, \vec{s})$ where $\vec{s}$ denotes the nucleon polarization vector in its rest frame. $T_{00}(r)$ and $T_{0 k}(\vec{r}, \vec{s})$ yield the energy and angular momentum densities which are obviously normalized: integrating them over space yields respectively the mass and the spin of the particle. With $\vec{e}=\vec{r} / r, r=|\vec{r}|$ the stress tensor (for spin-0 or spin- $-\frac{1}{2}$ ) is given by

$$
T_{i j}(\vec{r})=s(r)\left(e_{i} e_{j}-\frac{1}{3} \delta_{i j}\right)+p(r) \delta_{i j},
$$

where $p(r), s(r)$ denote the pressure and shear force distributions. EMT conservation $\partial^{\mu} \hat{T}_{\mu \nu}=0$ implies for the static stress tensor $\nabla^{i} T_{i j}(\vec{r})=0$. This in turn implies that $p(r)$ and $s(r)$ satisfy

$$
\frac{2}{3} \frac{\partial s(r)}{\partial r}+\frac{2 s(r)}{r}+\frac{\partial p(r)}{\partial r}=0 .
$$

It moreover implies the von Laue condition [39], a necessary (not sufficient) condition for stability,

$$
\int_{0}^{\infty} \mathrm{d} r r^{2} p(r)=0 .
$$

The $D$-term can be expressed in two different ways in terms of shear and pressure distributions as

$$
D \stackrel{(a)}{=} m \int \mathrm{d}^{3} r r^{2} p(r) \stackrel{(\mathrm{b})}{=}-\frac{4}{15} m \int \mathrm{d}^{3} r r^{2} s(r) .
$$

Due to mechanical stability arguments we expect the densities to comply with the constraints [38]

$$
\text { (a) } T_{00}(r) \geq 0, \quad \text { (b) } \frac{2}{3} s(r)+p(r) \geq 0 \text {. }
$$




\section{Klein-Gordon particles and Goldstone bosons}

The first $D$-term calculation, of a scalar boson in free Klein-Gordon theory, was reported in [1]. Defining the two EMT form factors of a scalar particle analog to Eq. (1.1) one finds $D=-1$ [14]. One finds $D=-1$ also for the Goldstone bosons of spontaneous chiral symmetry breaking in the soft-pion limit [15, 16], which can also be derived from a soft-pion theorem for pion GPDs [10]. Chiral corrections up to $\mathscr{O}\left(E^{4}\right)$ (with $E$ denoting masses or momenta) to EMT form factors were computed for real world pions, kaons and $\eta$-mesons in chiral perturbation theory $[17,18]$. It is customary to assign an "internal size" to these hadrons through the electric form factor of e.g. $\pi^{+}$ according to $F(t)=1+\frac{1}{6} t\left\langle r^{2}\right\rangle_{\pi}+\mathscr{O}\left(t^{2}\right)$. However, pions, kaons and even $\eta$-mesons are too light to discuss 3D densities [14]. The situation is different for nuclei [14], which we shall discuss next.

\section{Nuclei}

In [13] also first insights into the physics of $D$-terms of nuclei were given: assuming nuclei (in the liquid drop model) to have "sharp edges" pressure and shear forces are given by $p(r)=p_{0} \theta\left(R_{n}-r\right)-\frac{1}{3} p_{0} R_{n} \delta\left(R_{n}-r\right)$ and $s(r)=\gamma \delta\left(R_{n}-r\right)$ with surface tension $\gamma=\frac{1}{2} p_{0} R_{n}$. This yields for the $D$-term

$$
D_{\text {nucleus }}=-\frac{4}{5}\left(\frac{4 \pi}{3}\right) m_{n} \gamma R_{n}^{4} \text {. }
$$

Finite skin-effects make the $\Theta$ - and $\delta$-functions in $p(r)$ and $s(r)$ smooth, see Fig. 1 , and the $D$-term more negative. Remarkably $D_{\text {nucleus }} \propto A^{7 / 3}$ since the nuclear masses and radii grow like $m_{n} \propto A$ and $R_{n} \propto A^{1 / 3}$ with the mass number [13]. Calculations in more sophisticated nuclear models support this prediction [28].

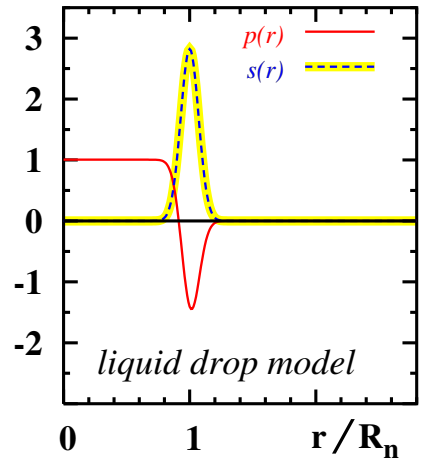

Figure 1: The pressure and shear forces of nuclei (in units of $p_{0}$ ) as functions of $r$ (in units of nuclear radius $R_{n}$ ) in the liquid drop model.

\section{Nucleon}

Also the $D$-term of the nucleon is negative [22]. Fig. 2 shows predictions for the pressure from the chiral quark-soliton model [24]: $p(r)$ is positive for $r<r_{0}$, and negative for $r>r_{0}$ with $r_{0} \simeq(0.5-0.6) \mathrm{fm}$. Positive $p(r)$ in the inner region means repulsion. This is intuitively interpreted as the "Fermi pressure" due to "Pauli blocking" in the quark core. Negative pressure in the outer region means attraction which is associated with the chiral soliton fields responsible for binding the quarks. The repulsive and attractive forces balance each other exactly according to the von Laue condition (3.3), as shown in Fig. 2a. This multiplied by $r^{2}$ (and by the prefactor $4 \pi M_{N}$ with nucleon mass $M_{N}$ ) yields the integrand of $D$ in Eq. (3.4a) depicted in Fig. 2b which ultimately yields a negative $D$. In early works it was therefore conjectured that the negative sign of the $D$-term is a consequence of stability [24]. This is not incorrect, but not the full story as we shall see later. In the chiral limit $p(r)$ and $s(r)$ behave like $1 / r^{6}$ at large distances. Therefore $D$ exists in the chiral limit, but $\left.D^{\prime}(t)\right|_{t=0} \propto 1 / m_{\pi}$ diverges [24]. The predictions from the chiral quark soliton model were shown [25] to be in good agreement with available lattice QCD results [26]. 

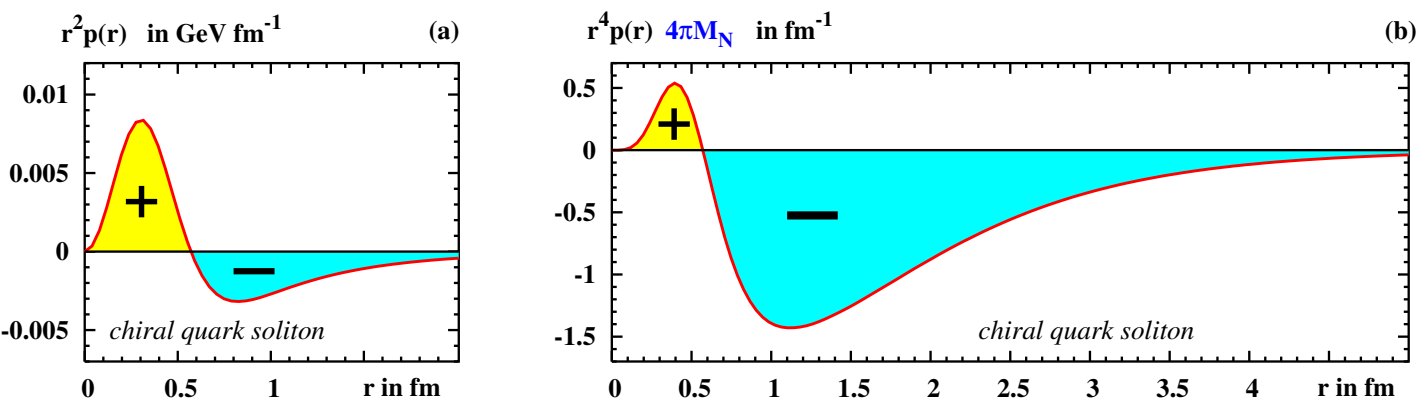

Figure 2: The pressure distribution inside nucleon from chiral quark soliton model [24]. (a) the shaded areas of $r^{2} p(r)$ above and below $y$-axis are exactly equal to each other demonstrating how the von Laue condition is realized. (b) $r^{4} p(r)$ is (up to a prefactor) the integrand of the $D$-term demonstrating why $D<0$.

\section{Lessons from $Q$-balls and $Q$-clouds}

$Q$-balls are non-topological solitons in theories with global symmetries [40]. They might have formed in the early universe and are dark matter candidates. $Q$-balls were also used to gain insights on the $D$-term in scalar theories $[34,35,36,37]$ where solitons are of the type $\Phi(t, \vec{r})=e^{i \omega t} \phi(r)$ for $\omega_{\min }<\omega<\omega_{\max }$ with the limits determined by details of the theory [40].

For $\omega \rightarrow \omega_{\min }$ one deals with $Q$-balls [40], see Fig. 3, absolutely stable solutions which share features of liquid drops and have $D<0$ [34]. Interestingly, meta-stable and unstable $Q$-balls [34] and their excitations [35] have also $D<0$. It is therefore fair to say that stability implies a negative $D$-term. But the reverse does need not be true: a negative $D$ does not necessarily imply stability.

For $\omega \rightarrow \omega_{\max }$ one deals with $Q$-clouds [41], unstable solutions which delocalize, spread out over all space, and form an infinitely dilute system of free quanta. Defining $\varepsilon^{2}=\omega_{\max }^{2}-\omega^{2}$ one finds that at fixed $r$ energy density $T_{00}(r)$ is diluted as $\varepsilon^{2}$. At the same time $\phi(r) \propto e^{-\varepsilon r}$ at large $r$, and the spatial size of the solutions diverges as $1 / \varepsilon$. As a consequence mass and $D$-term diverge as $M \sim 1 / \varepsilon$ and $D \sim 1 / \varepsilon^{2}$. Could this so extremely unstable and singular system have positive $D$ ? The answer is no! By carefully taming the divergences one finds that quantities like $\varepsilon M$ and $\varepsilon^{2} D$ have well-defined limits for $\varepsilon \rightarrow 0$, and the $D$-term expressed in these units is negative [36].

If even such extreme instabilities as the $Q$-cloud have negative $D$-terms, the question emerges whether it is possible to encounter a consistent system, however unstable it may be, which exhibits a positive $D$-term. No physical system with a positive $D$-term is known so far, except for an artifact in rigid rotator approach to the description of baryons in large $N_{c}$ limit discussed in the next section.
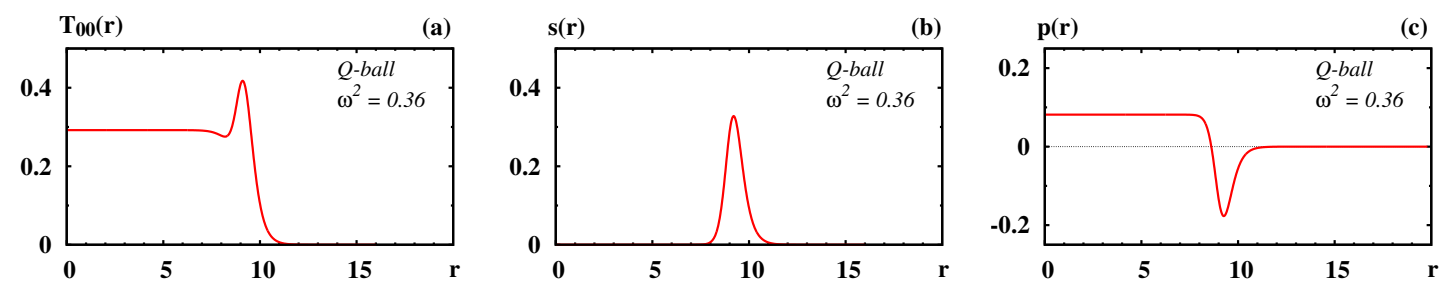

Figure 3: The energy distribution $T_{00}(r)$, shear forces $s(r)$ and pressure $p(r)$ inside a stable $Q$-ball [34]. 


\section{8. $\Delta$-resonance and unobserved states of the rigid rotator}

A similar situation is encountered with $\Delta$. It is an unstable resonance, but nevertheless it has a negative $D$-term as shown recently in a Skyrme model study [38]. Soliton models based on the large- $N_{c}$ expansion, such as the chiral quark-soliton model or Skyrme model, describe light baryons with spin and isospin quantum numbers $S=I=\frac{1}{2}, \frac{3}{2}, \frac{5}{2}, \ldots$ as different rotational states of the same soliton solution. In this "rigid rotator approach" one considers $1 / N_{c}$ corrections by expanding the action in terms of the slow angular velocity of rotating solitons. In contrast to the quantum numbers $S=I=\frac{1}{2}$ and $\frac{3}{2}$ corresponding to the nucleon and $\Delta$, one does not observe states with $S=I \geq \frac{5}{2}$ in nature. For a long time this unsatisfactory artifact of the rigid rotator approach was not understood. The issue was clarified in [38]. Working in the Skyrme model it was shown that the $1 / N_{c}$ corrections constitute a small perturbation in the nucleon case $S=I=\frac{1}{2}$. The corrections are more sizable for $S=I=\frac{3}{2}$ but one still obtains a consistent description of the $\Delta$ with $D<0$. However, for faster rotating solitons $S=I \geq \frac{5}{2}$ the $1 / N_{c}$ corrections become so destabilizing, that the basic stability criterion (3.5b) is violated. The results are shown in Fig. 4.

The energy density does not hint at anything unusual, see Fig. 4a: the states become heavier with increasing spin which makes sense. The shear forces are more insightful. All systems studied so far exhibit $s(r) \geq 0$, and this is also the case for $S=I=\frac{1}{2}, \frac{3}{2}$ whereby $s(r)$ becomes broader for $S=\frac{3}{2}$ indicating that $\Delta$ is even more diffuse than the nucleon. But for $S=I \geq \frac{5}{2}$ the shear forces become negative, see Fig. 4b, and one obtains a similarly reversed picture for the pressure which is negative in the center and positive in the outer region, see Fig. 4c. Also this has not been found in any other previous calculation. Both features imply at once a positive $D$-term. Even though $p(r)$ still complies with the von Laue condition (3.3), one deals for $S=I \geq \frac{5}{2}$ with an unstable equilibrium. The slightest disturbance of such a system makes it explode: matter is expelled from the center and dispersed to infinity. The stability criterion (3.5b) is violated, see Fig. $4 \mathrm{~d}$.

This study does not provide an example for a physical system with a positive $D$-term. It rather shows that these solutions are unphysical, explaining why the quantum numbers $S=I \geq \frac{5}{2}$ are indeed artifacts of the rigid rotator approach and not observed in nature [38].
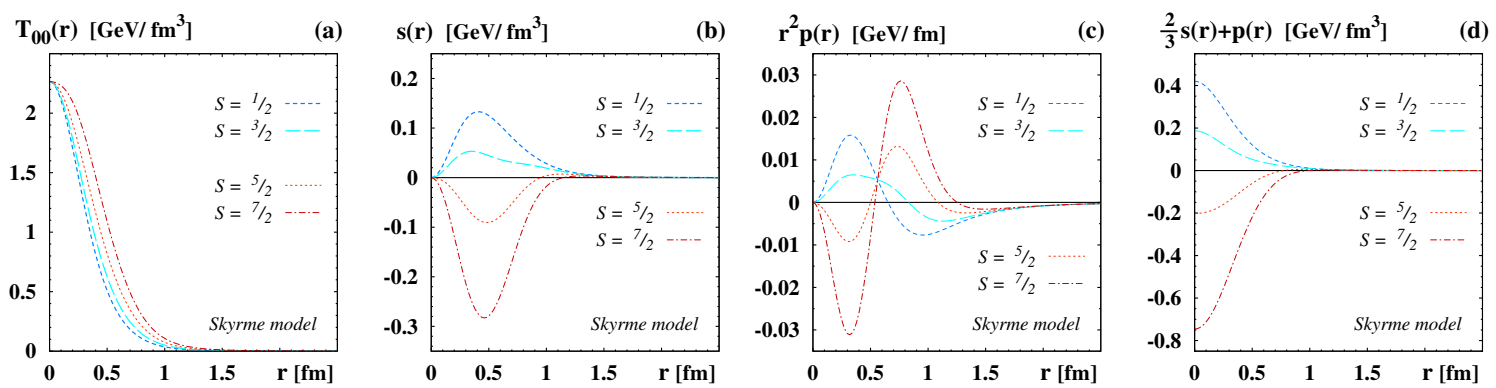

Figure 4: EMT densities in Skyrme model for the quantum numbers $S=I=\frac{1}{2}, \frac{3}{2}, \frac{5}{2}, \frac{7}{2}$. Energy density $T_{00}(r)$ indicates the states are heavier with increasing spin, and does not reveal anything unusual. More insightful are $s(r)$ and $p(r)$ which flip signs and violated the stability criterion $\frac{2}{3} s(r)+p(r) \geq 0$ for $S=I \geq \frac{5}{2}$. This calculation explains why $S=I=\frac{1}{2}, \frac{3}{2}$ are physical states corresponding to nucleon and $\Delta$. In contract to this $S=I \geq \frac{5}{2}$ are unphysical artifacts of the rigid rotator approach which are not observed in nature [38]. 


\section{Application to hidden-charm pentaquark $P_{c}(4450)$ at $\mathbf{L H C b}$}

A practical application of EMT densities was presented recently in [42], namely to pentaquark states observed by LHCb in $\Lambda_{b}^{0} \rightarrow J / \Psi p K^{-}$[43]. Most of the time one observes weak decays $\Lambda_{b}^{0} \rightarrow J / \Psi \Lambda^{*}$ followed by strong decays $\Lambda^{*} \rightarrow p K^{-}$. But the $J / \Psi p$ spectrum contains also structures compatible with pentaquark $P_{c}^{+}(c \bar{c} u u d)$ resonances. In about $(8.4 \pm 0.7 \pm 4.2) \%$ of the cases a broad resonance $P_{c}^{+}(4380)$, and in about $(4.1 \pm 0.5 \pm 1.1) \%$ of the cases a narrow resonance $P_{c}^{+}(4450)$ is formed. These observations cannot be explained in terms of $K^{-} p$ resonant or nonresonant contributions, and are compatible with analyses of $\Lambda_{b}^{0} \rightarrow J / \psi p \pi^{-}$decays [44].

One way to describe such states was proposed in [42]: the narrow $P_{c}^{+}(4450)$ can be interpreted as an $s$-wave nucleon- $\psi(2 S)$ bound state with $J^{P}=\frac{3}{2}-$ (these quantum numbers are not "most preferred" by the analysis [43], but also compatible with the data). The binding mechanism is due to an effective charmonium-nucleon interaction [45] given in terms of the charmonium chromoelectric polarizability and nucleon EMT densities. In Ref. [42] also a $J^{P}=\frac{1}{2}^{-}$state was predicted with nearly the same mass as $P_{c}^{+}(4450)$ modulo hyperfine splitting effects which are suppressed as $1 / m_{Q}$ in heavy quark mass limit. The broader resonance $P_{c}(4380)$ does not appear as a nucleon$\psi(2 S)$ bound state in [42]. There are also no nucleon- $J / \Psi$ bound states, as the effective interaction is too weak in this channel. In the original study [42] the EMT predictions were taken from the chiral quark soliton model [24], and the results were confirmed in [38] using Skyrme model [29]. The description of $P_{c}^{+}(4450)$ as a $\psi(2 S)$-nucleon bound state is therefore rather robust.

In [38] also model-independent lower bounds for chromoelectric polarizabilities of quarkonia were derived which show that charmonium-baryon bound states can exist for a variety of baryons. As an application bound states in the $\Delta-\psi(2 S)$ channel were predicted: a narrower negative-parity $s$-wave bound state in the around $4.5 \mathrm{GeV}$ with width around $70 \mathrm{MeV}$, and a broader positive-parity $p$-wave resonance around $4.9 \mathrm{GeV}$ with width of $\mathscr{O}(150 \mathrm{MeV})$. Both actually come in a family of spin states $J=\frac{1}{2}, \frac{3}{2}, \frac{5}{2}$ from combining spins of $\psi(2 S)$ and $\Delta$ with small mass-splittings due to hyperfine quarkonium-baryon spin-spin interactions. These new states $P_{\Delta c}$ could be observed in weak decays of bottom-baryons $\Lambda_{b}^{0}, \Sigma_{b}, \Xi_{b}$ or in photon-nucleon or pion-nucleon reactions. This prediction will allow an important test of the approach. Also charmonium-hyperon bound states may exist [38].
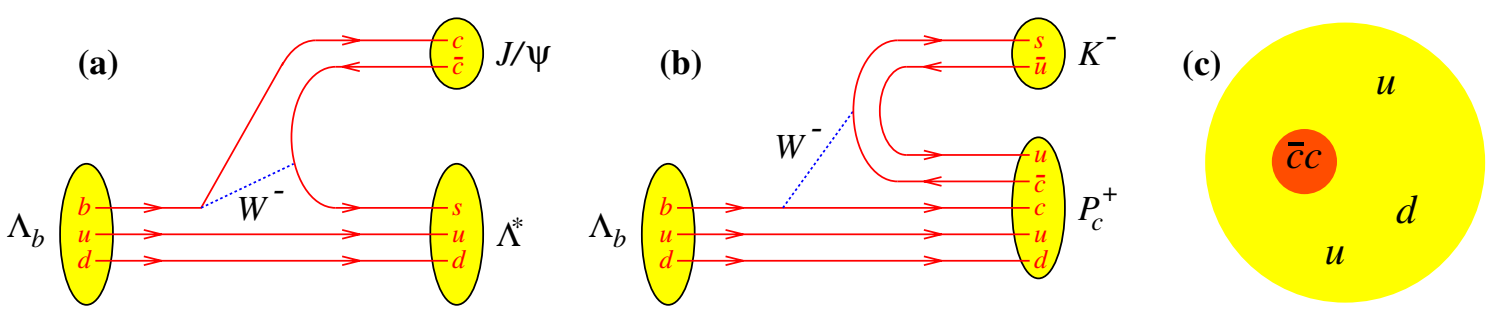

Figure 5: Mechanisms of $\Lambda_{b}^{0} \rightarrow J / \Psi p K^{-}$decays studied at LHCb [43]. (a) The dominant reaction is the weak decay $\Lambda_{b}^{0} \rightarrow J / \Psi \Lambda^{*}$ followed by the strong decay $\Lambda^{*} \rightarrow p K^{-}$. (b) A significant fraction of the events proceeds via the formation of $K^{-}$and exotic resonances $P_{c}^{+}$followed by subsequent $P_{c}^{+} \rightarrow J / \psi p$ decays. (c) The charmonium-baryon bound states arise because the small $c \bar{c}$ penetrate the baryon, and are bound due to an effective potential which can be expressed in terms of EMT densities [42, 38]. 


\section{Summary and Outlook}

Some aspects of the EMT structure, especially regarding the spin- or mass-decomposition of the nucleon, gained certain prominence in literature. But the prospective uses of another highly interesting direction remain largely unexplored: the stress tensor and the $D$-term.

Studies of the stress tensor and $D$-term in a variety of theoretical approaches - from soft pion theorems, to models, to lattice $\mathrm{QCD}$, to $Q$-balls, to dispersion relations - indicate the rich potential of this field. Among the promising new insights is the perspective to learn about the distribution of strong forces inside the nucleon and nuclei. This will shed valuable light on how the internal strong forces balance to form hadronic states [13].

Currently the EMT structure and the $D$-term of the nucleon are not known experimentally. It will be a long way before we will know them. However, preliminary (and at the current stage of art necessarily model-dependent) projections indicate that information about the $D$-term and the pressure distribution of the nucleon can be mapped out through measurements of hard-exclusive reactions at Jefferson Lab [46] or COMPASS. The experiments are in preparation.

An exciting development is that the knowledge of the EMT may play an important role in the description of hidden-charm pentaquarks observed at LHCb [42]. Further studies are underway and experimental tests are needed. But the fascinating development sketched in Fig. 6 is not unrealistic: the knowledge gained from studies of exclusive reactions at relatively modest energies at Jefferson Lab and COMPASS may constrain the dynamics of hidden-charm pentaquarks observed at LHCb. These exciting developments are worthwhile exploring and deserve further attention.

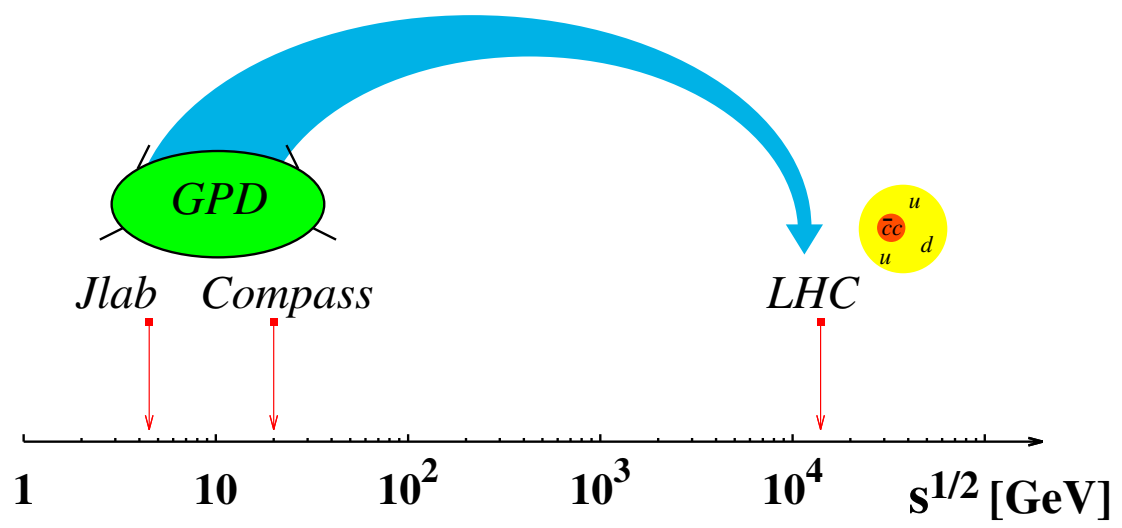

Figure 6: Information extracted from hard-exclusive processes at relatively modest energies via GPDs will help to constrain EMT form factors whose knowledge will enable us to calculate the effective quarkoniumbaryon interaction potentially allowing us to describe hidden-charm pentaquark states at LHCb.

\section{Acknowledgments}

P.S. would like to thank the organizers for creating the opportunity to present this research, and for local support. This work was supported in part by the National Science Foundation (Contract No. 1406298), and the Deutsche Forschungsgemeinschaft (Grant VO 1049/1). 


\section{References}

[1] H. R. Pagels, Phys. Rev. 144, 1250 (1965).

[2] D. Müller, D. Robaschik, B. Geyer, F. M. Dittes and J. Hořejši, Fortsch. Phys. 42, 101 (1994).

[3] A. V. Radyushkin, Phys. Lett. B 380, 417 and 385, 333 (1996); Phys. Rev. D 55, 7114 and 56, 5524 (1997).

[4] X. D. Ji, Phys. Rev. Lett. 78, 610 (1997).

[5] J. C. Collins, L. Frankfurt and M. Strikman, Phys. Rev. D 56, 2982 (1997).

[6] K. Goeke, M. V. Polyakov and M. Vanderhaeghen, Prog. Part. Nucl. Phys. 47, 401 (2001).

[7] X. D. Ji, J. Phys. G 24, 1181 (1998). A. V. Radyushkin, arXiv:hep-ph/0101225. M. Diehl, Phys. Rept. 388, 41 (2003). A. V. Belitsky and A. V. Radyushkin, Phys. Rept. 418, 1 (2005). M. Guidal, H. Moutarde and M. Vanderhaeghen, Rept. Prog. Phys. 76, 066202 (2013).

[8] X. D. Ji, Phys. Rev. Lett. 74, 1071 (1995); Phys. Rev. D 52, 271 (1995).

[9] “The Proton Mass: At the heart of most visible matter," workshop at Temple University, March 2016.

[10] M. V. Polyakov and C. Weiss, Phys. Rev. D 60, 114017 (1999).

[11] O. V. Teryaev, Phys. Lett. B 510, 125 (2001).

[12] R. G. Sachs, Phys. Rev. 126, 2256 (1962).

[13] M. V. Polyakov, Phys. Lett. B 555, 57 (2003).

[14] Jonathan Hudson and Peter Schweitzer, work in progress.

[15] V. A. Novikov and M. A. Shifman, Z. Phys. C 8, 43 (1981).

[16] M. B. Voloshin and V. I. Zakharov, Phys. Rev. Lett. 45, 688 (1980).

[17] J. F. Donoghue and H. Leutwyler, Z. Phys. C 52, 343 (1991).

[18] B. Kubis and U. G. Meissner, Nucl. Phys. A 671, 332 (2000) [Erratum-ibid. A 692, 647 (2001)].

[19] E. Megias, E. Ruiz Arriola, L. L. Salcedo and W. Broniowski, Phys. Rev. D 70, 034031 (2004).

E. Megias, E. Ruiz Arriola and L. L. Salcedo, Phys. Rev. D 72, 014001 (2005).

W. Broniowski and E. R. Arriola, Phys. Rev. D 78, 094011 (2008).

[20] J. W. Chen and X. D. Ji, Phys. Rev. Lett. 88, 052003 (2002).

A. V. Belitsky and X. D. Ji, Phys. Lett. B 538, 289 (2002).

S.-I. Ando, J.-W. Chen and C.-W. Kao, Phys. Rev. D 74, 094013 (2006).

M. Diehl, A. Manashov and A. Schäfer, Eur. Phys. J. A 29, 315 (2006).

[21] X. D. Ji, W. Melnitchouk and X. Song, Phys. Rev. D 56, 5511 (1997).

[22] V. Y. Petrov, P. V. Pobylitsa, M. V. Polyakov, I. Börnig, K. Goeke and C. Weiss, Phys. Rev. D 57, 4325 (1998).

[23] P. Schweitzer, S. Boffi and M. Radici, Phys. Rev. D 66, 114004 (2002).

J. Ossmann, M. V. Polyakov, P. Schweitzer, D. Urbano, K. Goeke, Phys. Rev. D 71, 034011 (2005).

M. Wakamatsu, Phys. Lett. B 648, 181 (2007).

[24] K. Goeke, J. Grabis, J. Ossmann, M. V. Polyakov, P. Schweitzer, A. Silva and D. Urbano, Phys. Rev. D 75, 094021 (2007). 
[25] K. Goeke, J. Grabis, J. Ossmann, P. Schweitzer, A. Silva and D. Urbano, Phys. Rev. C 75, 055207 (2007).

[26] P. Hägler et al. [LHPC collaboration], Phys. Rev. D 68, 034505 (2003) and 77, 094502 (2008). M. Göckeler et al. [QCDSF Collaboration], Phys. Rev. Lett. 92, 042002 (2004). J. D. Bratt et al., Phys. Rev. D 82, 094502 (2010). D. Brommel et al., PoS LAT 2005, 360 (2006).

[27] S. Liuti and S. K. Taneja, Phys. Rev. C 72, 032201 (2005).

[28] V. Guzey and M. Siddikov, J. Phys. G 32, 251 (2006).

[29] C. Cebulla, K. Goeke, J. Ossmann and P. Schweitzer, Nucl. Phys. A 794, 87 (2007).

J. H. Jung, U. Yakhshiev and H. C. Kim, J. Phys. G 41, 055107 (2014).

[30] H. C. Kim, P. Schweitzer and U. Yakhshiev, Phys. Lett. B 718, 625 (2012).

J. H. Jung, U. Yakhshiev, H. C. Kim and P. Schweitzer, Phys. Rev. D 89, 114021 (2014).

[31] B. Pasquini, M. V. Polyakov and M. Vanderhaeghen, Phys. Lett. B 739, 133 (2014).

[32] H. D. Son and H. C. Kim, Phys. Rev. D 90, 111901 (2014).

[33] I. R. Gabdrakhmanov and O. V. Teryaev, Phys. Lett. B 716, 417 (2012).

[34] M. Mai and P. Schweitzer, Phys. Rev. D 86, 076001 (2012).

[35] M. Mai and P. Schweitzer, Phys. Rev. D 86, 096002 (2012).

[36] M. Cantara, M. Mai and P. Schweitzer, Nucl. Phys. A 953, 1 (2016).

[37] F. Bergabo et al, talk at APS April Meeting 2017, Washington, DC, and work in progress.

[38] I. A. Perevalova, M. V. Polyakov and P. Schweitzer, Phys. Rev. D 94, 054024 (2016).

[39] M. von Laue, Ann. Phys. (Leipzig) 340, 524 (1911).

I. Białynicki-Birula, Phys. Lett. A 182, 346 (1993).

[40] S. R. Coleman, Nucl. Phys. B 262, 263 (1985) [Erratum-ibid. B 269, 744 (1986)].

[41] M. G. Alford, Nucl. Phys. B 298, 323 (1988).

[42] M. I. Eides, V. Y. Petrov and M. V. Polyakov, Phys. Rev. D 93, 054039 (2016).

[43] R. Aaij et al. [LHCb Collaboration], Phys. Rev. Lett. 115, 072001 (2015).

[44] R. Aaij et al. [LHCb Collaboration], Phys. Rev. Lett. 117, 082002 and 082003 (2016).

[45] M. B. Voloshin, Sov. J. Nucl. Phys. 36, 143 (1982) [Yad. Fiz. 36, 247 (1982)]; Prog. Part. Nucl. Phys. 61, 455 (2008).

[46] V. Burkert, plenary talk at SPIN 2016 in Urbana-Champaign, September 25-30, 2016. JLab Experiment PR12-16-010 "DVCS with CLAS12 at 6.6 GeV and $8.8 \mathrm{GeV}$." 